\title{
Is mainstream LCA linear?
}

\section{Reinout Heijungs ${ }^{1,2}$ [D}

Received: 10 March 2020 / Accepted: 12 August 2020 / Published online: 21 August 2020

(C) The Author(s) 2020

\begin{abstract}
Purpose It is frequently mentioned in literature that LCA is linear, without a proof, or even without a clear definition of the criterion for linearity. Here we study the meaning of the term linear, and in relation to that, the question if LCA is indeed linear. Methods We explore the different meanings of the term linearity in the context of mathematical models. This leads to a distinction between linear functions, homogeneous functions, homogenous linear functions, bilinear functions, and multilinear functions. Each of them is defined in accessible terms and illustrated with examples.

Results We analyze traditional, matrix-based, LCA, and conclude that LCA is not linear in any of the senses defined.

Discussion and conclusions Despite the negative answer to the research question, there are many respects in which LCA can be regarded to be, at least to some extent, linear. We discuss a few of such cases. We also discuss a few practical implications for practitioners of LCA and for developers of new methods for LCI and LCIA.
\end{abstract}

Keywords LCA $\cdot$ Life cycle assessment $\cdot$ Linear models $\cdot$ Nonlinear models $\cdot$ Linearity

\section{Purpose}

We often read statements concerning the linearity of LCA. As an example, below are a few quotations from articles in The International Journal of Life Cycle Assessment:

- "Linear models such as... LCA" (Yang 2017; Yang and Heijungs 2018)

- "At the heart of LCA methodology, default assumptions lie in the strict linearity between inputs and outputs" (Gibon and Schaubroeck 2017)

- "Unlike conventional LCA, [our] life cycle inventory... relies on complex nonlinear calculations" (Di Lullo et al. 2020)

- "The reduction of model uncertainties in the inventory analysis through the use of nonlinear modeling" (Huijbregts 1998)

Communicated by: Yi Yang

Reinout Heijungs

r.heijungs@vu.nl

1 Department of Supply Chain Analytics, Vrije Universiteit Amsterdam, De Boelelaan 1105, 1081

HV Amsterdam, The Netherlands

2 Institute of Environmental Sciences, Leiden University, PO Box 9518, Leiden 2300 RA, The Netherlands
- "The LCA model scaled linearly" (Villares et al. 2017)

- "The first adaptation necessary for LCA... is to permit nonlinear relationships in the inventory models" (Geyer et al. 2010)

- "LCA, in effect, assumes linearity of all concentrationresponse functions: all impact indices are proportional to the emitted quantities" (Spadaro and Rabl 1999)

Apparently, many authors seem to agree that mainstream LCA, or at least mainstream LCI, is linear. However, saying that LCA is linear is one thing, but it is another how such authors arrive at that conclusion, and what they mean by it.

In this paper, we will study two questions:

- Question 1: what does it mean when we say that LCA is linear?

- Question 2: is it true that mainstream LCA is indeed linear?

Answers to these two questions may induce new questions, such as the following:

- Is it bad that LCA is linear?

- Can we develop improvements to cure such problems?

- When is a linear model good enough? 
Such follow-up questions will be left to future contributions; here we only discuss the two questions about the meaning of linearity and if mainstream LCA is linear. Despite this seemingly narrow scope of the research, there appears to be a surprisingly rich and nuanced answer to the apparently simple four-word question "Is mainstream LCA linear?".

In posing the question if LCA is linear, there is a third question which needs to be answered: What do we mean by LCA? Yes, we mean environmental life cycle assessment, but even then there are a large number of variations: processbased LCA, IO-based LCA, consequential LCA, attributional LCA, dynamic LCA, etc. To avoid the danger of forgetting very specialized forms of LCA, we will narrow the discussion to mainstream LCA. This is admittedly not clearly defined as well, but we think that everyone who regularly reads The International Journal of Life Cycle Assessment and who has used the usual databases and software for LCA knows what we have in mind. Computationally, it relies on (1) the scaling of unit processes (or IO sectors) such that a final demand (typically, the functional unit) is satisfied, (2) the aggregation of similar pollutants and resources over all life cycle processes or sectors, and (3) the use of characterization factors in LCIA. The LCI part is often expressed in matrix form (Heijungs and Suh 2002), although variations also occur (Ciroth et al. 2004). The LCIA part is generally done with equations such as $\sum_{i} C$ $F_{i} \times m_{i}$ (Hauschild et al. 2018) or matrix equivalents (Rosenbaum et al. 2007).

\section{Methods}

Words like "linear" and "linearity" have different meanings. We can speak about a "linear chain of events" or a "linear narrative": First, I drop water on the floor, then a passerby slides and falls, then the person breaks his leg, then he goes to hospital, then he sues me, and then... This narrative meaning is not completely alien to LCA; consider for instance the phrase "the circular economy is a move from linear business models... to circular business models" (Broadbent 2016), where the word "linear" is used in such a way. Another pertinent example is the contrast between the cradle-to-grave "linear" LCA and "circular" cradle-to-cradle frameworks: "McDonough and Braungart... recognized the importance of closing "technical" and "biological" loops in a "cradle-to-cradle" or circular (rather than cradle-tograve or linear) economy" (Bocken et al. 2016). A third case is the recognition that in the ISO framework "a number of arrows indicate that rather than a linearly proceeding process, LCA involves many feedback loops between the different phases of the LCA" (Hauschild et al. 2018).
However, in most cases, the term "linear" is used in a different, more mathematical, way. Below, we will study the meaning of linearity in the context of mathematical models.

\subsection{Linear, homogeneous, homogeneous linear, bilinear, and multilinear functions}

Typically, where authors write about the linearity (or nonlinearity) of LCA, they refer to a certain property, which has to do with straight lines in a graph and/or proportionality of response ("twice as much product means twice as much emission"). For instance, Yang (2017) writes that "when we apply such linear models as process- and IO-based LCA to address change-related questions, they are based on a strict linear or proportional relationship". We will focus on such uses, bringing in a bit more precision through mathematical definitions and mathematical notation. In this analysis, we will base ourselves on the standard textbooks on mathematics, such as Apostol (1967), Adams and Essex (2010), and Stewart (2012).

A function $f(x)$ is said to be a linear function when it can be written as $a+b x$. For example, $f_{1}(x)=5+x$ and $f_{2}(x)=\frac{1}{2} x$ are linear functions, but $f_{3}(x)=3+\frac{2}{x-2}$ and $f_{4}(x)=-2+x^{2}$ are nonlinear. The graph of a linear function is a straight line. Figure 1 illustrates the four functions on a small part of their domain.

Note that the straight line that represents a linear function does not necessarily pass through the point $(0,0)$. This implies that there is not necessarily a proportionality between input $(x)$ and output $(f(x))$. For example, $f_{1}(x)=5+x$ is a linear function, but $f_{1}(2 \times 3)=11 \neq 2 \times f_{1}(3)=16$, so doubling input does not result in a doubled output. The subset of linear functions that satisfy the proportionality between input and output are sometimes referred to as homogeneous linear functions: A

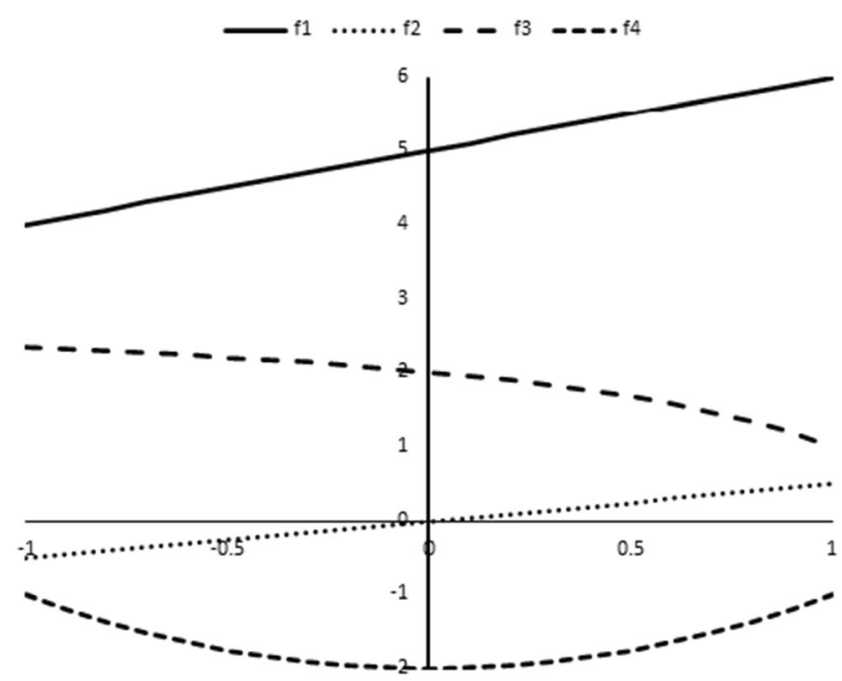

Fig. 1 Plot of two linear functions $\left(f_{1}(x)=5+x\right.$ and $\left.f_{2}(x)=\frac{1}{2} x\right)$ and two nonlinear functions $\left(f_{3}(x)=3+\frac{2}{x-2}\right.$ and $\left.f_{4}(x)=-2+x^{2}\right)$ 
homogeneous linear function satisfies $f(k x)=k f(x)$ for all values of $x$ and $k$. The subset of linear functions that are not homogeneous (so functions of the type $f(x)=a+b x$ with $a \neq$ 0 ) are then called non-homogeneous linear functions.

Besides functions of one variable, like $f(x)=a+b x$, mathematical theory also has incorporated functions of two or more variables, such as $g(x, y)=a+b x+c y$ or $h(x, y, z)=a+b x+$ $c y+d z$. Such functions also fall within the definition of linear functions. The essential thing is that all terms contain only one variable at the same time, and that this variable is included with order 1 , so without any square, root, logarithm, or other nonlinear ways. Thus, $g_{1}(x, y)=4-2 x+5 y$ and $g_{2}(x, y)=x-$ $y$ are linear functions, while $g_{3}(x, y)=4+x^{2}-5 y$ and $g_{4}(x$, $y)=3-4 x+2 x y$ are nonlinear. While linear functions of one variable can be plotted as a straight line in a 2-dimensional plot (Fig. 1), linear functions of two variables can be represented as a plane in a 3-dimensional graph (Fig. 2, left panel). With a bit of phantasy, linear functions of $k$ variables can be plotted as hyperplanes in a $(k+1)$-dimensional graph.

The proportionality between input and output is of course more complicated for functions of more than one variable. The concept of homogeneous linear functions works out in a slightly more complicated way because we should rescale all variables with the same factor: $g(k x, k x)=k g(x, y)$ for a function of two variables, $h(k x, k x, k z)=k h(x, y, z)$ for a function of three variables, etc. More generally, if a linear function $f$ satisfies $f(k x, k y, k z, \ldots)=k f(x, y, z, \ldots)$ for all $k$, it is a homogeneous linear function.

A function of two or more variables that is nonlinear can still be homogeneous. An example is the function $g(x, y)=\frac{8 x y}{x+y}$. Clearly, $g(k x, k y)=\frac{k^{2}}{k} \frac{8 x y}{x+y}=k g(x, y)$, for all $k \neq 0$. This function therefore satisfies a proportionality feature (doubling both $x$ and $y$ yields a doubled output), but it is clearly not a linear function (Fig. 2, right panel).

An important change of perspective occurs when we consider functions such as $g(x, y)=a+b x+c y+d x y$. This function is not linear, in the sense as defined above: it is a nonlinear function. However, when we fix one of the variables (say, $x$ ) and change the other variable $(y)$, the response changes in a linear way. To see this, we rewrite the function as $g_{x}(y)=(a+$ $b x)+(c+d x) y$, which indeed looks like a linear function of one variable (only $y$ ). If we plot $g_{x}$ against $y$, for a fixed $x$, we will see a straight line with slope $c+d x$. Such functions are referred to as bilinear functions (Fig. 3). The idea of studying the effect of a change while keeping the other variable fixed points of course to the partial derivative: $\frac{\partial g}{\partial y}=c+d x$, which does not depend on $x$. Likewise, $\frac{\partial g}{\partial x}=b+d y$ does not depend on $y$. Note that every linear function of two variable is also a bilinear function $\left(g(x, y)=a+b x+c y\right.$ yields $\frac{\partial g}{\partial x}=b$ and $\frac{\partial g}{\partial y}=c$ ), but that the converse does not hold $(g(x, y)=a+b x+c y+$ $d x y$ cannot be written as $\left.g(x, y)=a^{\prime}+b^{\prime} x+c^{\prime} y\right)$.

In the more general case of more than two variables, we speak of multilinear functions: A function $h(x, y, \ldots)$ is a multilinear function when $\frac{\partial h}{\partial x}$ does not depend on $x$, $\frac{\partial h}{\partial y}$ does not depend on $y$, and similar relations hold for all other variables. In the remaining text, we will no longer mention bilinearity, but always group this under multilinearity. Table 1 summarizes the different concepts.

As a final note, a function like $g(x, y)=a+b x+c y^{2}$ is a nonlinear function because there is a quadratic term in it. It is also non-multilinear, because $\frac{\partial g}{\partial y}=2 c y$, which depends on $y$. However, it can be considered to be linear (and multilinear) in $x$ (but still nonlinear in $y$ ). In mathematics, linearity and multilinearity are absolute notions, and a function that is nonlinear in one of its variables is nonlinear. However, we will here allow for such cases of "partial" linearity or multilinearity. Please note that the terms partial linearity and partial multilinearity are not generally recognized terms in mathematics.

\subsection{Question 1: what does it mean when we say that LCA is linear?}

On the basis of the mathematical definitions of linear, homogeneous linear, and multilinear functions, we can answer the first question as follows: LCA depends on a lot of data. More specifically, we mention several categories of data:

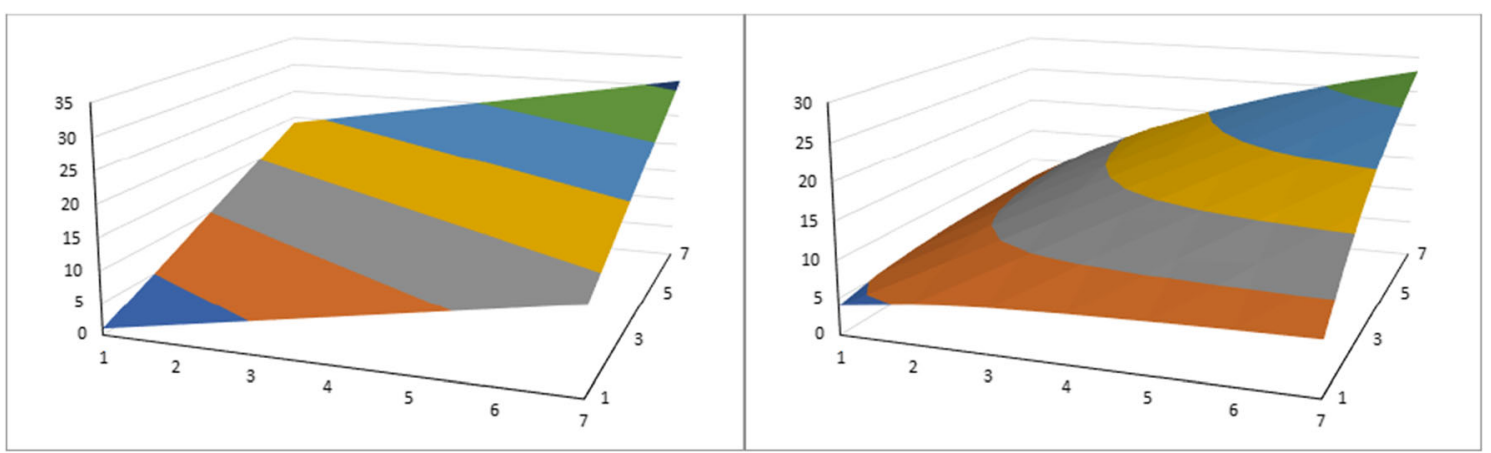

Fig. 2 Left panel: plot of a linear function of two variables $(g(x, y)=3 x+2 y-4)$. Right panel: plot of a homogeneous nonlinear function $\left(g(x, y)=\frac{8 x y}{x+y}\right)$. In both panels, the colors indicate different function value ranges (e.g., orange is between 5 and 10) 


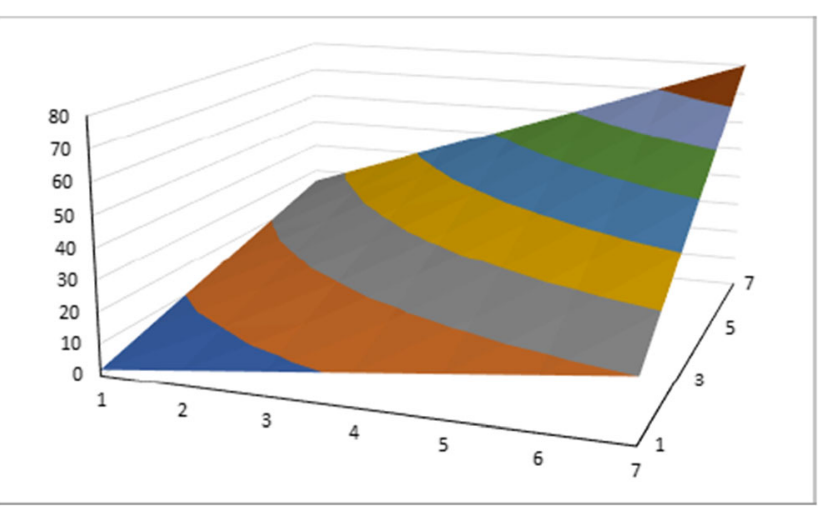

Fig. 3 Plot of a bilinear (but not linear) function of two variables ( $g(x$, $y)=3 x+2 y+x y-4)$. The colors indicate different function value ranges (e.g., orange is between 10 and 20)

- Goal and scope definition: the functional unit/reference flow;

- Inventory analysis: product flows (e.g., electricity use per unit of steel);

- Inventory analysis: elementary flows (e.g., $\mathrm{CO}_{2}$ emission per unit of electricity);

- Impact assessment: characterization factors (e.g., the GWP of methane);

- Impact assessment: normalization factors and weighting factors.

There may be some more data needed, for instance in relation to allocation factors. We will ignore these here.

We now propose the following answer to question 1 :

- LCA is linear if the LCA result is a linear function of the data that is used as input in the LCA calculations.

Here, LCA result may refer to the result of inventory, characterization, normalization, or weighting. In addition, we allow for possible other types of nuances:
- LCA is homogenous linear if the LCA result is a homogenous linear function of the data that is used as input in the LCA calculations;

- LCA is homogenous if the LCA result is a homogenous function of the data that is used as input in the LCA calculations;

- LCA is multilinear if the LCA result is a multilinear function of the data that is used as input in the LCA calculations.

\section{Results}

To study the question if LCA is linear (or homogenous, or homogeneous linear, or multilinear), we will consider an LCA system of minimal size:

- Two unit processes;

- Two products

- One elementary flow

- Only LCI, no impact assessment.

The next subsection describes the setup of this system and specifies the final model equation.

\subsection{The model equation of a small LCA system}

We will work with traditional matrix-based setup (Heijungs and Suh 2002), which is based on linear (sic!) algebra. Here, we have a final demand vector $\mathbf{f}$ $=\left(\begin{array}{ll}f_{1} & f_{2}\end{array}\right)$, a technology matrix $\mathbf{A}=\left(\begin{array}{lll}a_{11} & a_{12} & a_{21} a_{22}\end{array}\right)$, and an intervention matrix which is reduced to a row vector $\mathbf{B}=\left(\begin{array}{lll}b_{11} & b_{12}\end{array}\right)$. The result is the system-wide

Table 1 Classification of a number of functions of 1, 2 or 3 variables in terms of linearity, homogeneity, homogeneous linearity, and multilinearity. Bilinearity is multilinearity in the case of 2 variables

\begin{tabular}{|c|c|c|c|c|c|}
\hline Variables & Example function & Linear & Homogeneous linear & Homogeneous & $\begin{array}{l}\text { Bilinear/ } \\
\text { multilinear }\end{array}$ \\
\hline 1 & $f(x)=a x$ & Yes & Yes & Yes & Yes \\
\hline 1 & $f(x)=a+b x$ & Yes & No & No & Yes \\
\hline 1 & $f(x)=a+b x^{2}$ & No & No & No & No \\
\hline 2 & $g(x, y)=a x+b y$ & Yes & Yes & No & Yes \\
\hline 2 & $g(x, y)=a+b x+c y$ & Yes & No & No & Yes \\
\hline 2 & $g(x, y)=a+b x^{2}+c y$ & No & No & No & No \\
\hline 2 & $g(x, y)=a+b x+c y+d x y$ & No & No & No & Yes \\
\hline 2 & $g(x, y)=a+b x+c y+d x y+e x^{2}$ & No & No & No & No \\
\hline 2 & $g(x, y)=a x+a y$ & Yes & Yes & Yes & Yes \\
\hline 2 & $g(x, y)=\frac{a x y}{b x+b y}$ & No & No & Yes & No \\
\hline 3 & $h(x, y, z)=a+b x+c y+d z$ & Yes & No & No & Yes \\
\hline
\end{tabular}


emission vector (g), for which we use the generic formula

$$
\mathbf{g}=\mathbf{B A}^{-1} \mathbf{f}
$$

For such a 2-by-2-system, the inverse of $\mathbf{A}$ can be worked out in algebraic detail:

$$
\mathbf{A}^{-1}=\frac{1}{a_{11} a_{22}-a_{12} a_{21}}\left(\begin{array}{cc}
a_{22} & -a_{12} \\
-a_{21} & a_{11}
\end{array}\right)
$$

This then yields

$g_{1}=\frac{b_{11} a_{22} f_{1}-b_{11} a_{12} f_{2}-b_{12} a_{21} f_{1}+b_{12} a_{11} f_{2}}{a_{11} a_{22}-a_{12} a_{21}}$

In summary, the LCI result $\left(g_{1}\right)$ is modeled as a function $F$ of the different inputs $\left(b_{11}, b_{12}, a_{11}, a_{12}, a_{21}, a_{22}, f_{1}, f_{2}\right)$ :

$g_{1}=F\left(b_{11}, b_{12}, a_{11}, a_{12}, a_{21}, a_{22}, f_{1}, f_{2}\right)$

The question we face now is as follows: Is the function $F$ linear, homogeneous linear, homogeneous, multilinear, or is it just nonlinear in all meanings?

\subsection{Question 2: is it true that mainstream LCA is indeed linear?}

We consider the different types of linearity of the function

$$
\begin{aligned}
& F\left(b_{11}, b_{12}, a_{11}, a_{12}, a_{21}, a_{22}, f_{1}, f_{2}\right) \\
& =\frac{b_{11} a_{22} f_{1}-b_{11} a_{12} f_{2}-b_{12} a_{21} f_{1}+b_{12} a_{11} f_{2}}{a_{11} a_{22}-a_{12} a_{21}}
\end{aligned}
$$

This function is clearly not of the type

$$
\begin{array}{r}
F\left(b_{11}, b_{12}, a_{11}, a_{12}, a_{21}, a_{22}, f_{1}, f_{2}\right) \\
=\alpha+\beta b_{11}+\gamma b_{12}+\delta a_{11}+\cdots
\end{array}
$$

so it is not a linear function. Because of this, it is also not a homogeneous linear function.

It is a homogeneous function, though:

$$
\begin{gathered}
F\left(k b_{11}, k b_{12}, k a_{11}, k a_{12}, k a_{21}, k a_{22}, k f_{1}, k f_{2}\right) \\
=k F\left(b_{11}, b_{12}, a_{11}, a_{12}, a_{21}, a_{22}, f_{1}, f_{2}\right)
\end{gathered}
$$

In words, if we multiply all coefficients in an LCA calculation (functional unit, process data) by a factor of 2 , the LCI result will be doubled. It is easy to see that, although we did not analyze the impact assessment here, inclusion of characterization factors changes this conclusion. Anyhow, we do not think that this nonlinear homogeneity is the property that most authors have in mind when they write that LCA is linear.

The only further option to consider is multilinearity. To test for that, we consider the eight partial derivatives $\frac{\partial F}{\partial b_{11}}, \frac{\partial F}{\partial b_{12}}, \frac{\partial F}{\partial a_{11}}$, etc. For conciseness, we will work out just three representative cases.

First we consider $\frac{\partial F}{\partial b_{11}}$ :

$\frac{\partial F}{\partial b_{11}}=\frac{a_{22} f_{1}-a_{12} f_{2}}{a_{11} a_{22}-a_{12} a_{21}}$

which does not depend on $b_{11}$, so this suggests multilinearity with respect to $b_{11}$ (and by analogy, with respect to $\left.b_{12}\right)$. Next consider $\frac{\partial F}{\partial f_{1}}$ :

$\frac{\partial F}{\partial f_{1}}=\frac{b_{11} a_{22}-b_{12} a_{21}}{a_{11} a_{22}-a_{12} a_{21}}$

which does not depend on $f_{1}$, so again no problem in maintaining multilinearity with respect to $f_{1}$ (and $f_{2}$ ). Finally, $\frac{\partial F}{\partial a_{11}}$ :

$\frac{\partial F}{\partial a_{11}}=\frac{b_{12} f_{2}}{a_{11} a_{22}-a_{12} a_{21}}-a_{22} \frac{b_{11} a_{22} f_{1}-b_{11} a_{12} f_{2}-b_{12} a_{21} f_{1}+b_{12} a_{11} f_{2}}{\left(a_{11} a_{22}-a_{12} a_{21}\right)^{2}}$

Clearly, this expression depends on $a_{11}$, and we therefore conclude that $F$ is not multilinear with respect to $a_{11}$. Similar expressions and conclusions can be drawn when we consider the other $3 a$-variables $a_{12}, a_{21}$, and $a_{22}$.

Because the LCA function $F$ is non-multilinear in some of its arguments, it is a non-multilinear function, even though it is multilinear in some of its other arguments, so the overall conclusion is that LCA is not a multilinear function.

Now, we have analyzed the four possible options that could be embraced by linearity: linear, homogeneous linear, homogeneous, and multilinear. Traditional, matrix-based, LCA has been demonstrated to be at most nonlinear homogeneous, but not linear, not homogeneous linear, and not multilinear. Therefore, there is not much in LCA that could be argued to be linear. Our overall conclusion is therefore the following: No, LCA is not linear.

\section{Discussion and conclusions}

Despite our negative answer to the question raised in the title, the discussion is not finished. For instance, why do so many authors claim that LCA is linear? We will approach this question from different sides.

\subsection{Traces of linearity in LCA}

The analysis of multilinearity leaves open a small but important option: The LCA function $F$ is multilinear (not linear) if we consider the elements of $\mathbf{f}$ and $\mathbf{B}$ only. A plot of the LCA result as a function of one specific element of $\mathbf{f}$ (say, $f_{1}$ ) or one specific element of $\mathbf{B}$ (say, $b_{11}$ ) shows a straight line (but not necessarily through the point $(0,0)$ ). 
There is one other special case to consider. In conventional LCA, the final demand vector $\mathbf{f}$ is entirely filled with zeros, except for the reference product that fulfills the functional unit. In our small example, we might write this as $\mathbf{f}=\left(f_{1}\right.$ 0 ). In that case, the previous equation for $F$ reduces to

$$
\begin{aligned}
F & \left(b_{11}, b_{12}, a_{11}, a_{12}, a_{21}, a_{22}, f_{1}\right)=\frac{b_{11} a_{22} f_{1}-b_{12} a_{21} f_{1}}{a_{11} a_{22}-a_{12} a_{21}} \\
= & \frac{b_{11} a_{22}-b_{21} a_{12}}{a_{11} a_{22}-a_{12} a_{21}} f_{1}
\end{aligned}
$$

This implies that $F$ is a homogeneous linear function when we only study the effect of $f_{1}$. Indeed, the LCA result $\left(g_{1}\right)$ scales linearly with the size of the functional unit $\left(f_{1}\right)$. We conjecture that this proportionality of $g$ on $f_{1}$ is meant by some LCA researchers whenever they speak of the "linearity of LCA". Such is indeed the case with Yang and Heijungs (2018): "the amount of impact change $(\Delta \mathbf{g})$ that is associated with a change in demand $[(\Delta \mathbf{f})]$ is a linear function of $[\Delta \mathbf{f}]$. We will refer to this as process-based LCA being a linear model." (notation has been changed to agree with this paper).

This is of course a very specific and restricted meaning of linearity. As shown, LCA in general is not linear, it is even not multilinear, and it is certainly not homogeneous linear.

As a side note, with a compound functional unit like $\mathbf{f}$ $=\left(f_{1} f_{2}\right)$, we have no linearity, but we do have a homogeneous case, because when we multiply all reference flows by the same factor $k$, so drive the system with $k \mathbf{f}=\left(k f_{1} k f_{2}\right)$, the LCA result also multiplies by $k$ into $k \mathbf{g}$. This is another trace of linearity, even though a mathematician would prefer to use the term "homogeneous" for this case.

\subsection{Almost linear LCA and strongly nonlinear LCA}

To better investigate the situation, we will choose concrete numbers for the input variables of our LCA system, based on Heijungs and Suh (2002) and Heijungs (2002):

$$
\mathbf{A}=\left(\begin{array}{cc}
-2 & 100 \\
10 & 0
\end{array}\right)
$$

and

$$
\mathbf{B}=\left(\begin{array}{ll}
1 & 10
\end{array}\right)
$$

and

$$
\mathbf{f}=\left(\begin{array}{c}
0 \\
1000
\end{array}\right)
$$

With these choices, we can compute a value for $g: 120$. If we now vary coefficient $a_{22}$ between 0 (the "default" value above) and -499 , we obtain a strongly nonlinear response (Fig. 4, left panel). If we zoom in on a smaller range of values, between 0 and -25 , the response is for all practical purposes linear (Fig. 4, right panel).

The consequence is that for some ranges of parameter values, the nonlinear LCA result responds in an almost linear way, while in some other ranges, the response is not at all linear.

\subsection{Causes of nonlinearity}

How can linear (sic!) algebra, which is at the root of matrixbased LCA (Heijungs and Suh 2002), lead to a nonlinear model? The reason is that the basic equation

$\mathbf{g}=\mathbf{B A}^{-1} \mathbf{f}$

looks like a succession of "innocent" multiplications, but in fact contains a crucial power $\left(^{-1}\right)$ for the inverse of matrix $\mathbf{A}$. In several respects, a matrix inversion bears similarities with a division, so we may in a way reinterpret $\mathbf{B} \mathbf{A}^{-1} \mathbf{f}$ as “ $\frac{\mathbf{B f}}{\mathbf{A}}$ ” (note this is an interpretation only, and it is not a division). As noted in the example function $f_{3}(x)=3+\frac{2}{x}$ in the very beginning of our analysis, division yields a nonlinear function (Fig. 1).

A related point is that the start equations of LCA are linear. They are based on equations like

$$
\left\{\begin{array}{l}
\mathbf{A s}=\mathbf{f} \\
\mathbf{g}=\mathbf{B s}
\end{array}\right.
$$

both of which are linear. The point is that $\mathbf{A s}=\mathbf{f}$ is a system of linear equations, but that its solution $\mathbf{s}=\mathbf{A}^{-\mathbf{1}} \mathbf{f}$ is nonlinear. This issue has been addressed in the gray LCA literature before (Heijungs (2002): "even though processes are scaled in LCA in a linear way, the final equation that displays how the $\mathrm{CO}_{2}$ emission depends on the system's coefficients is nonlinear."). In other words, part of the confusion about the alleged linear nature of LCA may stem from the distinction between the equations and the solution of the equations. Traditional LCA scales processes in a linear way, but the scaling factors depend in a nonlinear way on the process data.

The conclusion is that in statements like "LCA is a linear model" not only the term "linear" deserves a more precise articulation, but also the distinction between a model equation and the solution of the model equation should be introduced carefully. LCA's model equations are linear, but their solutions are nonlinear. The distinction between a model equation and the solution of a model equation is a fundamental one, but it is not always appreciated. In our case, the model $\mathbf{A s}=\mathbf{f}$ is linear, but its solution $\mathbf{s}=\mathbf{A}^{-1} \mathbf{f}$ is nonlinear, because it involves an inversion.

\subsection{Extension to LCIA}

The discussion above has concentrated on the inventory phase of LCA. Does inclusion of impact assessment (the LCIA phase) provide new insights? We will restrict the discussion 


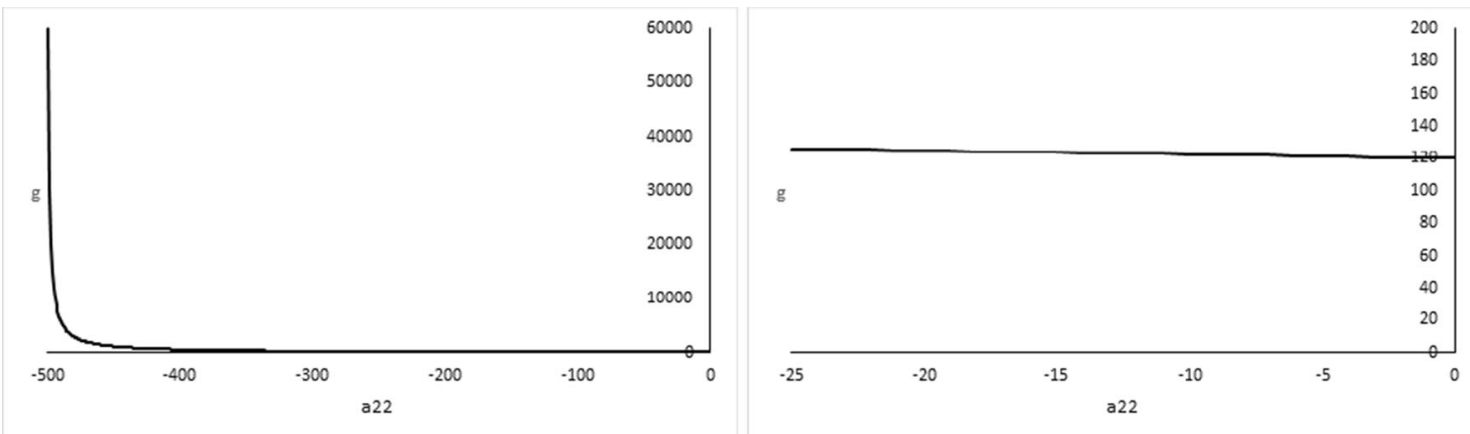

Fig. 4 Dependence of the LCA result $(g)$ on $a_{22}$. Left panel: over a large range of values, a nonlinear response is clearly visible. Right panel: over a small range of values, the response is approximately linear (but not homogeneous linear)

to characterization; extension to normalization or weighting is straightforward.

Traditional LCIA uses characterization factors, which are used to perform a linear aggregation of LCI results. A typical expression for the characterization result for impact category $j$, $h_{j}$, is

$h_{j}=\sum_{i} q_{j i} g_{i}$

where $q_{j i}$ is the characterization factor that couples elementary flow $i$ to impact category $j$. In matrix form, this may be written as

$\mathbf{h}=\mathbf{Q g}$

Clearly, this procedure satisfies the definition of a linear function (but not that of a homogeneous linear function).

The full LCA procedure can be written as

$$
\mathbf{h}=\mathbf{Q B A}^{-1} \mathbf{f}
$$

which of course remains nonlinear, as discussed above. But another intriguing argument may be added.

Characterization factors are typically derived from nonlinear models, such as the IPCC-climate model or species sensitivity distribution models for ecosystem toxicity. Let us write such a model as

$z=f(x, y)$

where $z$ is the model output (e.g., infrared radiation) and $x$ and $y$ are model inputs (e.g., the $\mathrm{CO}_{2}$ emission and the $\mathrm{CH}_{4}$ emission). Characterization factors are defined as marginal effects (Huijbregts et al. 2011), so through partial derivatives:

$q_{x}=\frac{\partial f}{\partial x}, q_{y}=\frac{\partial f}{\partial y}$

The LCIA result is then found through

$h=q_{x} g_{x}+q_{y} g_{y}$ where $g_{x}$ is the LCI result for elementary flow $x$ and $g_{y}$ is the LCI result for elementary flow $y$. With respect to the discussion on linearity, it is important to observe the following:

- Characterization factors are defined as partial derivatives from (in general) nonlinear impact models;

- Characterization factors are used in a linear way (the LCIA equation is linear).

As such, the use of characterization factors can be considered a "linearized" approach (Woods et al. 2018). Further, although some classes of characterization factors have historically been derived without the use of partial derivatives, it has even turned out to be possible to erect an underlying nonlinear impact model ex-post (Hélias and Heijungs 2019).

\subsection{Nonlinear LCA and parametrized LCA}

Although we concluded that LCA is in many respects nonlinear, the basic modeling principle of traditional LCA rests on a linearity assumption: Processes scale in a linear way. In the world of input-output analysis (Miller and Blair 2009), this assumption is known as the assumption of a linear technology. More precisely, it is an assumption of homogeneous linearity: For any process, doubling the input is associated with doubling the output. A specific process $j$ has exchanges with the economy $a_{i j}$ and with the environment $b_{k j}$. The linear relationship is then written as

$$
\begin{gathered}
a_{i j}^{\prime}=a_{i j} s_{j}(\text { for all } i) \\
b_{k j}^{\prime}=b_{k j} s_{j}(\text { for all } k)
\end{gathered}
$$

where $a_{i j}^{\prime}$ and $b_{k j}^{\prime}$ are the exchanges of process $j$ after scaling with a factor $s_{j}$ (while $a_{i j}$ and $b_{k j}$ are the exchanges per unit of output). This assumption of linear technology is made for every included process, after which the system-wide equations are the well-known 
$\left\{\begin{array}{l}\mathbf{f}=\mathbf{A s} \\ \mathbf{g}=\mathbf{B s}\end{array}\right.$

If we depart from the linearity assumption, we turn the equation for an individual process $j$ into a nonlinear function, say $\alpha_{i j}$ (for the economic part) and $\beta_{k j}$ (for the environmental part):

$a_{i j}^{\prime}=\alpha_{i j}\left(v_{j}\right)($ for all $i)$

$b_{k j}^{\prime}=\beta_{k j}\left(v_{j}\right)($ for all $k)$

where $v_{j}$ is some activity level (e.g., output level) of process $j$. The balance equation still holds:

$\sum_{j} \alpha_{i j}^{\prime}=\sum_{j} \alpha_{i j}\left(v_{j}\right)=f_{i}($ for all $i)$

The task is therefore to find the activity levels $v_{j}$ such that $\sum_{j} \alpha_{i j}\left(v_{j}\right)=f_{i}$, where $\alpha_{i j}$ are nonlinear functions. Depending on the details of these functions $\alpha_{i j}$, this may be a difficult task, and there is no guarantee that there is a solution at all, or a unique solution. In any case, there is no analogous closed-form solution, like $s_{j}=\sum_{i}\left(\mathbf{A}^{-1}\right)_{j i} f_{i}$ for the linear case.

Once these activity levels are found, they are inserted into

$g_{k}=\sum_{j} \beta_{k j}^{\prime}=\sum_{j} \beta_{k j}\left(v_{j}\right)($ for all $k)$

With $\beta_{k j}$ given, that is a straightforward task.

In conclusion, dropping the linear technology assumption in LCA is possible, but we have to replace the scaling factors $s_{j}$ (for which a closed-form solution is available) by activity levels $v_{j}$ (for which there is no closed-form solution).

The topic of parametrized LCA deserves a special mention. Parametrized LCA refers to a setup in which the process data (or some of the process data) depend collectively on one or more settings. For instance, the fuel efficiency of a car does not enter the process data of $\mathbf{A}$ and $\mathbf{B}$ explicitly, but it influences some of the elements in $\mathbf{A}$ and $\mathbf{B}$. The fuel input of process $j$ (say, $a_{i j}$ ) and the $\mathrm{CO}_{2}$ emission of process $j$ (say, $b_{k j}$ ) both depend on this external parameter (say, $\eta_{j}$ ). The dependence of these coefficients may even be nonlinear:

$a_{i j}=\phi_{i}\left(\eta_{j}\right), \quad b_{k j}=\psi_{k}\left(\eta_{j}\right)$

However, when the resulting process data $\left(a_{i j}\right.$ and $\left.b_{k j}\right)$ continue to end up in a matrix structure that is scaled in a linear way, the LCA model is again linear.

Notice, by the way, that the topic of parametrized is an underdeveloped one. Even a proper definition of what entails parametrized LCA is probably lacking, and different authors have different ideas on this. For instance, Mutel et al. (2013) suggest that parametrized LCA does not fit the framework of linear algebra: "the formulas... cannot be applied to parametrized databases, which include nonmatrix calculations". Marini and Blanc (2014), on the other hand, define "a parametrized LCA model [as] depending on a limited number of input parameter," and further treat it in the usual way. The whole idea of parametrized LCA obviously deserves a more rigorous treatment.

\subsection{The narrative meaning of linearity}

In the introduction, we briefly mentioned that the term linearity can be understood in a narrative way, besides the mathematical, model-oriented, meaning that has received our prime attention so far. LCA is sometimes considered to be linear, because it addresses a product from the cradle to the grave, as a chronologic account. Authors then sometimes argue that other paradigms (cradle-to-cradle, circular economy) have advantages above this linear thinking that would be inherent to LCA. This article will not further explore such debates. But we would like to single out one aspect: there are numerous loops in traditional LCA. For instance, electricity production requires fuel, while fuel production requires electricity. Such loops distort the linear time order of a flow diagram. Indeed, as Dorfman et al. (1958) observed in relation to input-output analysis, "for the production of coal, iron is required; for the production of iron, coal is required; no man can say whether the coal industry or the iron industry is earlier or later in the hierarchy of production". As a result, we may conceive the solution of the system to reflect some forms of time ordering, through the use of the power series expansion of the inverse matrix (Suh and Heijungs 2007):

$\mathbf{A}^{-1}=\mathbf{I}+(\mathbf{I}-\mathbf{A})+(\mathbf{I}-\mathbf{A})^{2}+(\mathbf{I}-\mathbf{A})^{3}+\cdots$

This is not further discussed here, but we refer to BeloinSaint-Pierre et al. (2014) for an elaboration.

A typical feature of such systems is that a network representation (Newman 2010) exhibits cyclic structures, and studying network theory opens another interesting vista for studying industrial networks (Heijungs 2015). Matrix-based LCA easily incorporates such structures in the model equations. In conclusion, there is no strict linear time order in LCA, and matrix-based LCA can easily deal with such "nonlinear" time orderings.

\subsection{Questions 1 and 2 once more}

The above arguments may lead to a more nuanced answer to question 1 (What does it mean when we say that LCA is linear?) and question 2 (Is it true that mainstream LCA is indeed linear?). Distinguishing the basic equations (such $\mathbf{A s}=\mathbf{f}$ ) from their solutions ( egies for answers: 
- Yes, the basic equations of (traditional) LCA are linear;

- Partly yes, the dependence of LCA results on the size of the functional unit data is linear;

- No, the dependence of LCA results on input data is nonlinear.

Likewise, we may answer:

- No, the dependence of LCA results on input data is nonlinear if you consider the whole range of values;

- Yes, the dependence of LCA results on input data is almost linear in a restricted range of values.

The strictly negative original answer to the title question (Is mainstream LCA linear?) can be relaxed if we adopt a more relaxed viewpoint of what it means to be "linear".

\subsection{Practical implications}

The analysis and discussion are quite theoretical so far. Do they also have practical implications, for present-day LCA, or for future development? Of course, there is an implication for the wording of research: simple statements that LCA is linear, such as those quoted in the introduction, have been shown to be incorrect or inaccurate. But there is a wider implication.

On the one hand, several authors criticize the use of "linear models" because they would fail to capture nonlinear effects, but then propose new, equally linear models. For example, Hauschild et al. (2018) write that "when medium-scale or large-scale interventions (or consequences) are to be assessed, the characterization factors should represent non-marginal potential impacts and may also have to consider nonlinearity". But at another place, they write that "the use of characterization models... implies a linear relationship," so it is unclear which type of nonlinearity problem would be resolved while staying within a linear (or multilinear) model setup. Especially in the world of LCIA method developers, there is an idea that traditional LCIA methods are of limited value due to a linear setup and that LCIA should move on to include nonlinear impact functions. For instance, Fantke et al. (2015) discuss that in an equation like $q \propto E R F$, where $q$ is an element of the characterization matrix $\mathbf{Q}$ and $E R F$ is the exposureresponse function, "a key assumption implicit in this framework is the linear, no-threshold $E R F$," and that "the shape of population $E R F$ is usually assumed to be linear with no threshold". That is wrong. As discussed by many authors (Huijbregts et al. 2011; Boulay et al. in press), characterization factors are derived from impact models that are either linear or nonlinear. That is, there is a dose-response function $f\left(x_{1}, x_{2}\right.$, $\ldots$ ), where $x_{i}$ is the emission (or concentration, or dose) of stressor $i$, and a marginal characterization factor is constructed through
$C F_{i}=\left.\frac{\partial f}{\partial x_{i}}\right|_{\left(x_{1}, x_{2}, \ldots\right)=\left(b_{1}, b_{2}, \ldots\right)}$

where $b_{i}$ is the background level of pollutant $i$ at which the derivative is determined (Heijungs and Suh 2002). If the effect would be zero below some threshold level, $C F$ would be zero at low levels until the background exceeds the threshold, after which $C F$ would be non-zero. Average characterization factors are instead made through

$C F_{i}=\frac{f\left(b_{1}, b_{2}, \ldots\right)}{b_{i}}$

This is admittedly a re-interpretation of the one-pollutant treatment by Huijbregts et al. (2011), who effectively use $\left.\frac{\partial f}{\partial x}\right|_{x=b}$ and $\frac{f(b)}{b}$ respectively, but we are not aware of any multi-pollutant presentation of the distinction between marginal and average characterization factor. If the impact function $f$ would be homogenous linear, the whole discussion on average versus marginal would be meaningless, because for such a function, $\frac{\partial f}{\partial x}=\frac{f(b)}{b}$ for all values of $x$ and $b$. Hélias and Heijungs (2019) discuss a few nonlinear impact models, and Cucurachi et al. (2012) present an operational example of a strongly nonlinear characterization model which is used to derive characterization factors. But indeed, LCIA linearizes such functions, because the CFs are invariably used in a characterization formula like

$h=\sum_{i} C F_{i} \times g_{i}$

Therefore, a possible point of improvement of LCIA would not be to replace linear impact models by nonlinear models, but to replace their linearized use through the concept of characterization factors by a more sophisticated system, replacing $\sum_{i} C F_{i} \times g_{i}$ by some nonlinear aggregation rule. As far as we know, that discussion has hardly taken place. The only place of which we know that this discussion is briefly mentioned is the multisubstance PAF treatment by Huijbregts et al. (2002), but unfortunately, no aggregation rule is stated by these authors, so it is impossible to say if their treatment is merely about a nonlinear $f$, or if it also includes a nonlinear use of characterization factors.

On the other hand, researchers should be well aware of the place at which linear and nonlinear considerations show up, and what this implies for proportionality. As was shown by Heijungs (2002), small changes of one LCA input coefficient may lead to huge changes in the LCA results, but in other cases, large changes of inputs may result in insignificant changes of results. The reason is that the LCA equations contain a mix of addition, multiplication, subtraction, and division, with non-trivial combined effects. A structured analysis of the sensitivity of the results to changes in the input 
coefficients is therefore needed. Quite a few LCA studies nowadays include an uncertainty analysis, but the use of sensitivity analysis is still far from standard practice. In that respect, not only global sensitivity (Padey et al. 2013; Groen et al. 2017) is important, but also local sensitivity (Wei et al. 2015; Senga Kiessé et al. 2017) should be taken seriously as well. Systematic study and reporting of such sensitivity analyses is still an exception rather than the rule.

\subsection{Conclusion}

Statements in literature on the linearity of LCA are mistaken. In the best case, LCA possesses a weaker property of multilinearity, but only with respects to elements of the final demand vector and the emission data. With respect to elements of the technology matrix, LCA is not multilinear, and certainly not linear, although the LCI is homogeneous. The strong property of homogeneous linearity is only present if we consider functional unit-based LCA as a function of the size of the functional unit. Only in that special case can we speak of a proportionality of the functional unit and the LCA result.

The underlying modeling principle in LCA is based on a linear technology assumption, where individual processes are assumed to be scalable in a linear homogeneous way. That by no means implies that the solution of the model equations is linear too. However, for certain ranges of parameter values, LCA results will behave in an almost linear way. That is obviously a poor reflection of reality, and it pinpoints to places in the LCA framework where better models are needed. For instance, the discussion on marginal or average linearization and (partial) derivatives (Huijbregts et al. 2011; Boulay et al. in press) is now primarily taking place in the LCIA, whereas it is equally essential in the LCI, where it is seldom discussed (see, however, Yang 2017). A better appreciation on what linearity embraces and how it enters the LCA model and the solution of the LCA model would help to clarify the discussion.

Acknowledgments Two anonymous reviewers added a number of interesting points that helped to better articulate the issue. The handling editor, Yi Yang, also supplied a few valuable points. I thank Thomas Schaubroeck for his remarks that stimulated me to write this article.

Open Access This article is licensed under a Creative Commons Attribution 4.0 International License, which permits use, sharing, adaptation, distribution and reproduction in any medium or format, as long as you give appropriate credit to the original author(s) and the source, provide a link to the Creative Commons licence, and indicate if changes were made. The images or other third party material in this article are included in the article's Creative Commons licence, unless indicated otherwise in a credit line to the material. If material is not included in the article's Creative Commons licence and your intended use is not permitted by statutory regulation or exceeds the permitted use, you will need to obtain permission directly from the copyright holder. To view a copy of this licence, visit http://creativecommons.org/licenses/by/4.0/.

\section{References}

Adams RA, Essex C (2010) Calculus. In: A complete course, 7th edn Pearson

Apostol TM (1967) Calculus. In: Volume I: one-variable calculus, with an introduction to linear algebra, 2nd edn. Wiley

Beloin-Saint-Pierre D, Heijungs R, Blanc I (2014) The ESPA (Enhanced Structural Path Analysis) method: a solution to an implementation challenge for dynamic life cycle assessment studies. Int J Life Cycle Assess 19:861-871

Bocken NMP, de Pauw I, Bakker C, van der Grinten B (2016) Product design and business model strategies for a circular economy. $\mathrm{J}$ Ind Prod Eng 33:308-320

Boulay A, Benini L, Sala S, in press. Marginal and non-marginal approaches in characterization: how context and scale affect the selection of an adequate characterization model. The AWARE model example. Int J Life Cycle Assess.

Broadbent C (2016) Steel's recyclability: demonstrating the benefits of recycling steel to achieve a circular economy. Int J Life Cycle Assess 21:1658-1665

Ciroth A, Fleischer G, Steinbach J (2004) Uncertainty calculation in life cycle assessments. A combined model of simulation and approximation. Int J Life Cycle Assess 9:216-226

Cucurachi S, Heijungs R, Ohlau K (2012) Towards a general framework for including noise impacts in LCA. Int J Life Cycle Assess 17:471487

Di Lullo G, Gemechu E, Oni AO, Kumar A (2020) Extending sensitivity analysis using regression to effectively disseminate life cycle assessment results. Int J Life Cycle Assess 25:222-239

Dorfman R, Samuelson PA, Solow RM 1958. Linear programming and economic analysis. Mc-Graw Hill.

Fantke P, Jolliet O, Evans JS, Apte JS, Cohen AJ, Hänninen OO, Hurley F, Jantunen MJ, Jerrett M, Levy JI, Loh MM, Marshall JD, Miller BG, Preiss P, Spadaro JV, Tainio M, Tuomisto JT, Weschler CJ, McKone TE (2015) Health effects of fine particulate matter in life cycle impact assessment: findings from the Basel Guidance Workshop. Int J Life Cycle Assess 20:276-288

Geyer R, Stoms DM, Lindner JP, Davis FW, Wittstock B (2010) Coupling GIS and LCA for biodiversity assessments of land use. Part 1: Inventory modeling. Int J Life Cycle Assess 15:454-467

Gibon T, Schaubroeck T (2017) Lifting the fog on characteristics and limitations of hybrid LCA - a reply to "Does hybrid LCA with a complete system boundary yield adequate results for product promotion?" by Yi Yang. Int J Life Cycle Assess 22(3):456-406. https://doi.org/10.1007/s11367-016-1256-9 Int J Life Cycle Assess 22, 1005-1008

Groen EA, Bokkers EAM, Heijungs R, De Boer IJM (2017) Methods for global sensitivity analysis in life cycle assessment. International Journal of Life Cycle Assessment 22:1125-1137

Hauschild MZ, Rosenbaum RK, Olsen SI (2018) Life cycle assessment. Springer, Theory and practice

Heijungs R 2002. The use of matrix perturbation theory for addressing sensitivity and uncertainty issues in LCA. p. 77-80. In: Anonymous (Ed.): Proceedings of the fifth international conference on ecobalance. Practical tools and thoughtful principles for sustainability. Nov. 6 - Nov. 8, 2002, Epochal Tsukuba, Tsukuba, Japan. Available at https://personal.vu.nl/R.Heijungs/docs/The\%20use\% 20 of $\% 20$ matrix $\% 20$ perturbation $\% 20$ the ory $\% 20$ for $\%$ 20addressing $\% 20$ sensitivity $\% 20$ and $\% 20$ uncertainty $\% 20$ issues $\%$ 20in\%20LCA\%20(2002).pdf.

Heijungs R (2015) Topological network theory and its application to LCA and related industrial ecology tools. J Env Acc Man 3:151-167

Heijungs R, Suh S (2002) The computational structure of life cycle assessment. Kluwer Academic Publishers 
Hélias A, Heijungs R (2019) Resource depletion potentials from bottomup models: population dynamics and the Hubbert peak theory. Sci Total Environ 650:1303-1308

Huijbregts MAJ (1998) Application of uncertainty and variability in LCA. Part I: A general framework for the analysis of uncertainty and variability in life cycle assessment. Int $\mathrm{J}$ Life Cycle Assess 3: 273-280

Huijbregts MAJ, Van de Meent D, Goedkoop M, Spriensma R (2002) Ecotoxicological impacts in life cycle assessment. In: Posthuma L, Suter GW II, Traas TP (eds) 2002Species sensitivity distributions in ecotoxicology. Lewis Publishers

Huijbregts M, Hellweg S, Hertwich E (2011) Do we need a paradigm shift in life cycle impact assessment? Environ Sci Technol 45:38333834

Marini C, Blanc I (2014) Towards prospective life cycle assessment: how to identify key parameters inducing most uncertainties in the future? Application to photovoltaic systems installed in Spain. In: Murgante $\mathrm{B}$ et al (eds) Computational science and its applications-ICCSA 2014. ICCSA 2014, Lecture Notes in Computer Science, vol 8581. Springer, Cham

Miller RE, Blair PD (2009) Input-output analysis. In: Foundations and extensions. Cambridge University Press

Mutel CL, de Baan L, Hellweg S (2013) Two-step sensitivity testing of parametrized and regionalized life cycle assessments: methodology and case study. Environ Sci Technol 47:5660-5667

Newman MEJ (2010) Networks. Oxford University Press, Oxford, An introduction

Padey P, Girard R, Le Boulch D, Blanc I (2013) From LCAs to simplified models: a generic methodology applied to wind power electricity. Environ Sci Technol 47:1231-1238

Rosenbaum RK, Margni M, Jolliet O (2007) A flexible matrix algebra framework for the multimedia multipathway modeling of emission to impacts. Environ Int 33:624-634

Senga Kiessé T, Ventura A, Van der Werf HMG, Cazacliu B, Idir R, Andrianandraina (2017) Introducing economic actors and their possibilities for action in LCA using sensitivity analysis: application to hemp-based insulation products for building applications. J Clean Prod 142:3905-3916

Spadaro JV, Rabl A (1999) Estimates of real damage from air pollution: site dependence and simple impact indices for LCA. Int J Life Cycle Assess 4:229-243

Stewart J (2012) Calculus. Seventh edition, Cengage

Suh S, Heijungs R (2007) Power series expansion and structural analysis for life cycle assessment. Int J Life Cycle Assess 12:381-390

Villares M, Iș1ldar A, van der Giesen C, Guinée J (2017) Does ex ante application enhance the usefulness of LCA? A case study on an emerging technology for metal recovery from e-waste. Int J Life Cycle Assess 22:1618-1633

Wei W, Larrey-Lassalle P, Faure T, Dumoulin N, Roux P, Mathias J-D (2015) How to conduct a proper sensitivity analysis in life cycle assessment: taking into account correlations within LCI data and interactions within the LCA calculation model. Environ Sci Technol 49:377-385

Woods JS, Damiani M, Fantke P, Henderson AD, Johnston JM, Bare J, Sala S, Maia de Souza D, Pfister S, Posthuma L, Rosenbaum RK, Verones F (2018) Ecosystem quality in LCIA: status quo, harmonization, and suggestions for the way forward. Int J Life Cycle Assess 23:1995-2006

Yang Y (2017) Rethinking system boundary in LCA — reply to "Lifting the fog on the characteristics and limitations of hybrid LCA" by Thomas Gibon and Thomas Schaubroeck (2017). Int J Life Cycle Assess 22:1009-1011

Yang Y, Heijungs R (2018) On the use of different models for consequential life cycle assessment. Int J Life Cycle Assess 23:751-758

Publisher's note Springer Nature remains neutral with regard to jurisdictional claims in published maps and institutional affiliations. 